\title{
First examinations with the newly installed posturographic platform at the University medical and dental center, Medical University - Varna, Bulgaria
}

\author{
Miroslav Stoykov', Stefan Peev', Hristina Arnautska', Christina Grupcheva², \\ Maria Stoeva², Daniela Petrova², Kalin Mihov³, Svetoslav Dobrilov³ \\ Ivan Angelov ${ }^{4}$, Mario Milkov' \\ 'University medical and dental center, Faculty of Dental medicine, Medical University - Varna \\ ${ }^{2}$ University Eye Hospital for active treatment, Medical University - Varna \\ ${ }^{3}$ Department of Orthopedics, University multiprofile hospital for active treatment "St. Marina" - Varna \\ ${ }^{4}$ Medical University - Varna
}

\section{Abstract}

Background

Posture of the human body aims to keep its balance when performing dynamic movements or in the static stance. Posturology is another term for the Global postural recalibration. It is the discipline which studies posture. Postural control is regulated by a complex system, known as the Tonic postural system. Stomatognathic system must not be excluded when discussing posture. Posturology enables clinicians to study the sensory information, entering the body and determine the sensor that work in a suboptimal manner.

Methods

A clinical case of a female patient, fully diagnosed, using the newly implemented system, is presented. Apart from it, we have analyzed another fourteen patients. All first filled out specifically designed questionnaires and signed written informed consent forms. Static and dynamic postural, audiological, vestibular, visual and HSAT assessments were performed, along with a dental check-up.

Results

Patient had normal occlusal characteristics - class 1 Angle, but problems with the temporomandibular joint and slight hearing were present. No vestibular, posture (static or dynamic) or visual convergence abnormalities were detected. Refractive abnormalities were present. Apart from that, a group of patients with obstructive sleep apnea is presented. Changes of their posture and balance were studied.

Conclusions

All information gathered by the two integrated static and dynamic analysis systems, along with the postural analyzer, HSAT and dental analysis can be used to define therapeutic interventions and exercise programmes needed.

Key words: static and dynamic posture analysis, SVV, vHIT, VNG, HSAT, dental occlusion

\section{Background}

Posture of the human body aims to keep its balance when performing dynamic movements or in the static stance. [1] Posture, which is automatic and unconscious, is stated as the position of the body in space. [2], [3], [11] It develops fully at the age of 11 and is stable up to the age of 65. [4], [13], [14] Posturology is another term for the Global postural recalibration. It is the discipline which studies posture. [5] Clinicians define it as a holistic therapy connected with the study of the tonic postural system. [6] It is already known that in order for the body to move, information from different sensors must be gathered. Those sensors are situated in the feet, eyes, jaw, inner ear. Information taken is sent to the brain for processing. The brain returns signals to the muscles for execution, so as minimal energy is used.

Postural control is regulated by a complex system, known as the Tonic postural system. Although complex it its nature, it is a simple reflex arc, where signals from visual system (retina), skin system (receptors in the feet), vestibular system (otoliths, transmitting information for acceleration and deviation of the head), and muscular system enter. [2] Stomatognathic system must not be excluded when discussing posture. Maxilla and mandibula, along with the masticatory muscles, extensor muscles of the neck, supra- and infrahyoid muscles and shoulder girdle, actively support the correct position of 
the skull. [5] It is proven that orthodontic changes of teeth are connected with changes in the normal posture of the skull and consequently - of the body. When all the specified sensors work properly and the head, shoulders, hips, feet are correctly positioned, authors speak of good posture (functional). When there are problems with those sensors, patients experience pain due to postural imbalance. In these cases, clinicians speak of bad posture (non-functional). [5], [6], [11], [15]

Posturology enables clinicians to study the sensory information, entering the body and determine the sensor that work in a suboptimal manner. Measures can be undertaken to address the imbalances. [7]

Balance is static and dynamic. In static situations, the body maintains static position. Spinal column forms the three physiological curvatures (cervical and lumbar lordosis, which convex forward, and dorsal kyphosis, which concaves forward), along the line of the center of gravity, aiding balance control. These curves form and stabilize at the age of 5 to 6 . The most important is the lumbar lordosis. [8], [9], [18]

In dynamic situations, the body maintains stability during daily activities. [5], [19], [20]

Main diagnostic technique, used to determine whether or not there is a postural pathology, is the method of stabilometry, referred sometimes as posturography. The patient stands on a platform - pictures of the posture of the body and the feet are taken, which are afterwards assessed. [10], [16], [17] Interdisciplinary teams of specialists, including otorhinolaryngologists, ophthalmologists, orthopedists, dentists, physiotherapists and chiropractors, podiatrists and others, can be formed to examine patients and provide care. Posture reeducation therapies rapidly gain popularity and recognition among experts. Many years of research now help clinicians to treat and prevent dysfunctions and injuries more effectively. Quality of life can be greatly improved. In November 2021, thanks to a project funded from the Bulgarian National Science Fund, won by a team of specialists at Medical University - Varna, a posturographic platform Global postural system PL0800 Leonardo (GPS, Chinesport) was delivered and installed. It consists of a central structure with a platform, a postural analyzer and three high-resolution pictures acquisition devices for static posture analysis on the frontal and sagittal plane. Dynamic and balance analysis is done with a bi-podalic support platform. This system allows non-invasive and painless examination of the patient's posture whenever it is deemed necessary. [12]

At the first stage of examination, the patient is invited to position on the postural analyzer for pictures acquisition. It is composed of two lateral aluminum rods, equipped with a measurement scale, an upper mirror with a midline, a plumb line (should be aligned with the patient malleolus), three horizontal and three vertical elastic stripes, which can be adjusted freely in order to determine asymmetries. The distance between the acquisition device and the person is predetermined and must be followed. Background for the pictures is recommended to be neutral and the same, so as the pictures to be standardized. In GPS Leonardo it is in the blue colour and provides adequate contrast. The upper mirror allows to diagnose possible shoulder rotations. Acquired four main images - front, rear, right, left, can be enlarged and allow the examiner to perform direct measurements on them, after calibration. For the exact patient position, the system is equipped with an adhesive coloured sticker with feet representation, allowing the patient to easily position. All taken images are saved in the patients' examination database profile. Images before and after treatment can be compared.

At the second stage of examination, going through the blue pathway, the patient goes to the dynamic posture and balance analyzer - the Podata platform. It is unique and original, allowing two levels of analysis. The first level of analysis allows acquiring real-size mono- or bi-podalic pictures of the patient's feet. The type of feet - normal, hollow or flat can be determined. At the second level of analysis, stabilometric data, through the six load cells, is gathered - gravity center of the examined patient and its position, compared to the ideal one, body weight distribution on the two feet. For each foot there are 3 reference points marked - on the first metatarsal, on the fifth metatarsal and on the heel (Kapandji reference). During the stabilometric tests, information about the front/rare/lateral axes fluctuation is collected - stabilogram, sway graphs (variations of the center of gravity in the set test and duration conditions), distribution of oscillation frequencies are then generated. Tests can be done in different stances - with open and closed eyes of the patient, with teeth clenched or not, with mouth open or not, with head rotated to the right or to the left, with the head inclined to the right or to the left. [12] 
The software allows the examiner to save and print a report with the patient's results, comparing different data acquired, in tables, graphs, diagrams.

Obstructive sleep apnea (OSA) is defined by the appearance of recurrent episodes of respiratory pauses (apneas) during sleep as a result of anatomical or functional obstacles in the upper respiratory tract. The possibility of airways to collapse and the expression of difficulties when breathing are increased. Secondary to the main disease, cognitive-behavioral, respiratory, cardiac, metabolic, and inflammatory disorders follow. [21] Hypoxia caused by OSA can alter and worsen balance control of the body. [22], [23], [24]

The visual system is one of the most involved system of the human organism when discussing the spatial orientation in the surroundings. It is known that proper binocular control can correct some of the mild dysfunctions in afferent systems. Stability during static standing involves maintaining the center of mass within the field of support. [28]

Changes in body posture are related to changes in head posture (including the lower jaw) and the cervical segment of the neck. The displacement of the head affects the visual field, the lordosis of the neck increases even more. [29], [30] Proper eye convergence and vestibular function, including balance and postural control, are two-way related- both convergence disorders affect balance and vestibular diseases affect vergence. [31], [32]

\section{Methods}

We present the first clinical case of a patient (female, aged 26), fully diagnosed, using the newly implemented system. She was first asked to fill out specifically designed questionnaires (dental and vestibular questions included) and to sign written informed consent. Then, static and dynamic postural, vestibular, audiological and visual assessments were done, along with a dental check-up (to determine aspects of occlusion) and Home Sleep apnea test (HSAT). We analyzed the posture and balance of fourteen patients with obstructive sleep apnea (OSA). The aim was to reach the connection between the posture and possible changes in it, the stomatognathic system (orthodontics) and the visual system, along with the implications on the vestibular system (balance control). Our target was as well to study in depth the aspects of the worsened balance control in patients with hearing problems and obstructive sleep apnea (OSA).

\section{Results}

In the first examined patient, we found:

\section{From the orthodontic clinical examination:}

- Sagittal relationship - Angle Class I Occlusion (frontal and distal regions, canines and molars, on the left and the right side), normal overjet

- Vertical relationship - normal overbite

- Transverse relationship - no changes in the middle line or the orthognathic relationships in the distal regions.

- No traumatic injuries as a child, except for a tendon sprain of the right thigh at the age of 13 .

- No other bad habits, except for chewing nails at the age period 4-7.

- Experiences pain in the left TMJ when fully opening the mouth and chewing nuts or other hard food, the joint luxates habitually.

- Upon closing the mandible after maximal opening, it closes with a S-movement to the right.

- No frequent headaches or muscle pain.

- Has undergone orthodontic treatment at the age of 14 , for a duration of 1.5 years.

\section{From the posturographic examinations:}
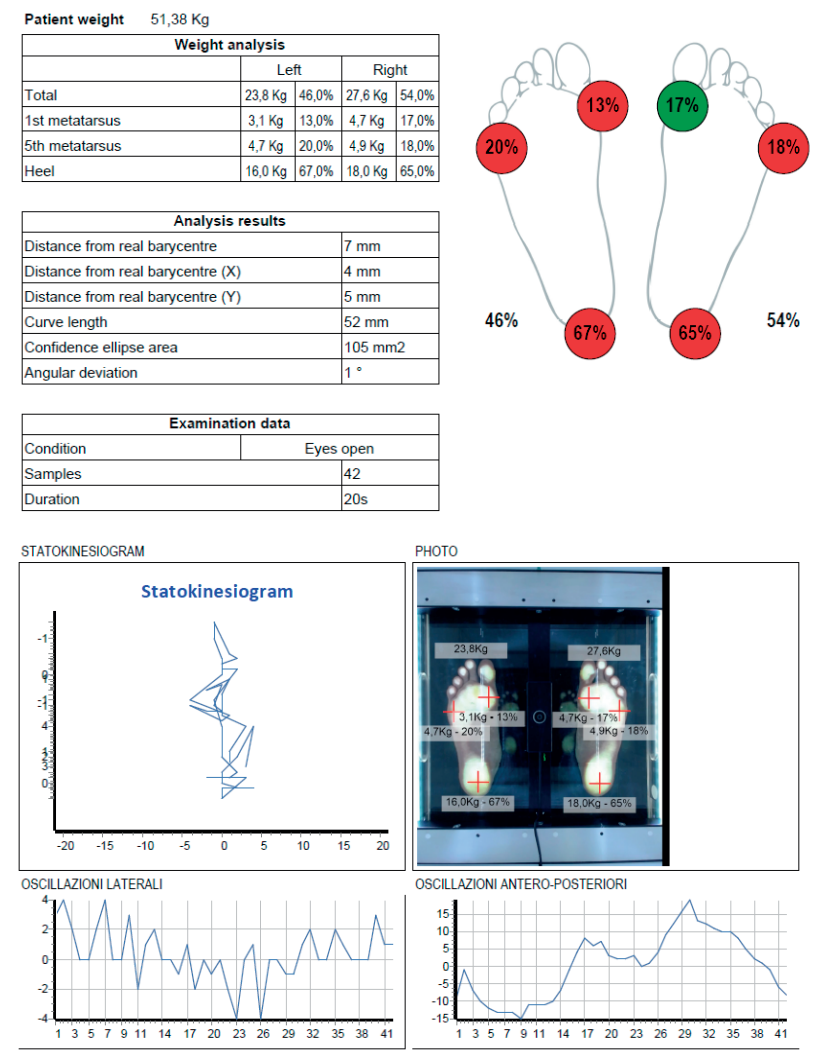

Fig. 1a, b. Stabilometric examination and weight distribution analysis, Eyes opened modality 

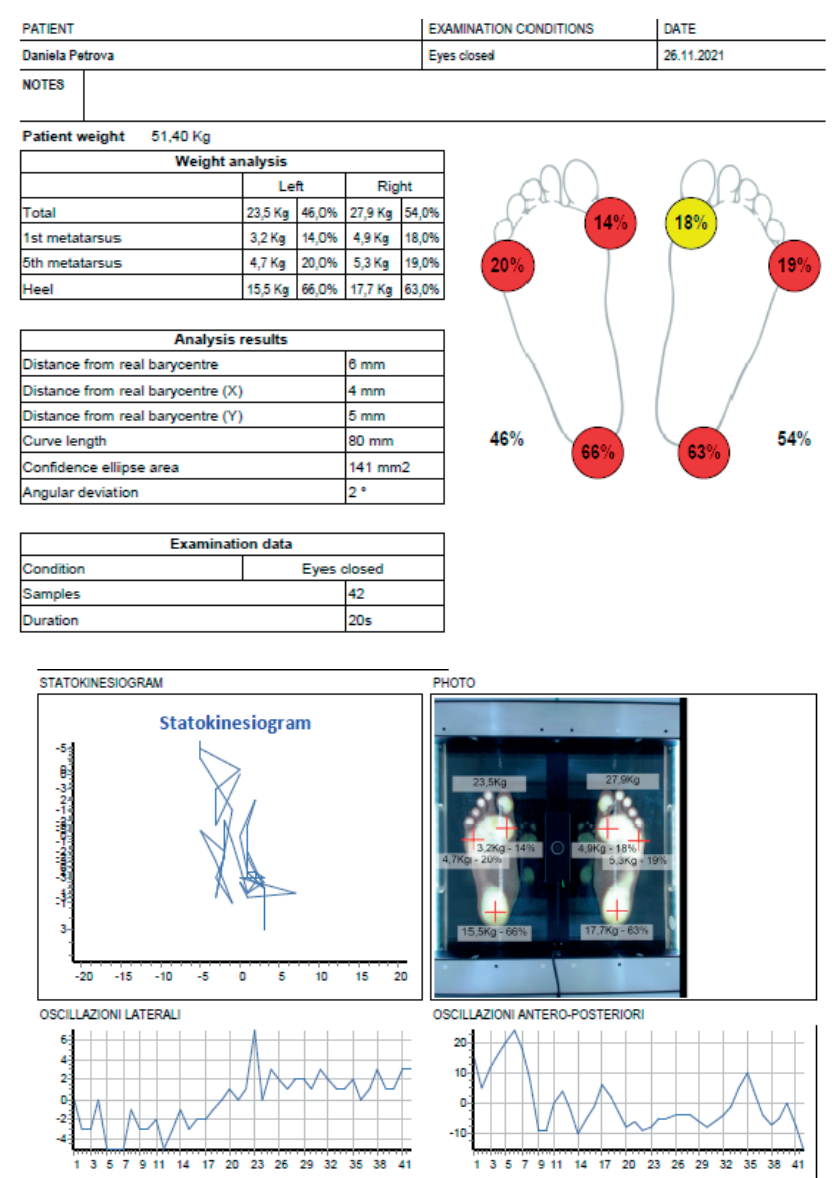

Fig. 2a, b. Stabilometric examination and weight distribution analysis, Eyes closed modality

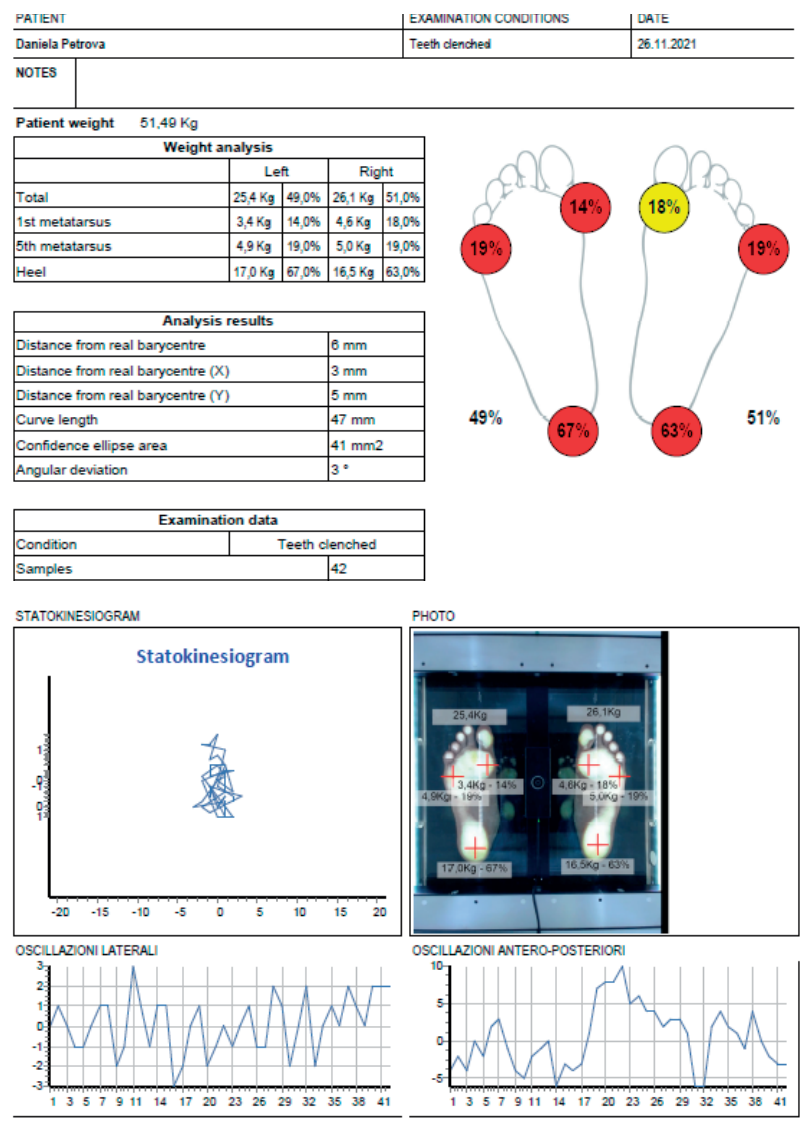

Fig. 3a, b. Stabilometric examination and weight distribution analysis, Teeth clenched modality

\section{From the vestibular examinations}

Patient underwent VNG (videonystagmography), vHIT (video Head impulse test), SVV (subjective visual vertical), Romberg, Fukuda examinations. No spontaneous nystagmus, nor pathological vestibulo-ocular reflexes from the vHIT-tests were detected. Romberg and Fukuda examinations were negative. Patient aligned correctly and without significant deviations the vertical line in the SVV-test.

\section{From the audiological examinations}

Slight diminishing in the hearing capability of the left ear was diagnosed.

\section{From the visual examinations}

Patient was diagnosed with hypermetropy and astigmatism: VOD: $+0.75 \mathrm{dsph} . /-2.50 \mathrm{dcyl}$ ax 4; VOS: $+1.25 \mathrm{dsph} /-2.50 \mathrm{dcyl}$ ax 171 . No abnormalities in convergence capabilities were found.

\section{From the HSAT}

AHI was determined 6, which is the border between no sleep apnea and a mild case of it.

\section{Patients with symptoms of OSA analyzed}

Fourteen patients (10 male and 4 female) with symptoms of OSA were analyzed. It was proven in all of them, more frequent in male individuals, consistent with another studies. [26], [27] Severe cases of obstructive sleep apnea were more frequent among male patients, consistent with the results from another studies on the topic. [25] Female patients tend to be left often underdiagnosed, because of not complaining of the symptoms of the disease and due to symptoms of other disorders. Problems with balance were more frequent in the female patients, which was proven by measuring the distance from the real barycenter of the body - all four $(100 \%)$ female patients with OSA had distances from the ideal barycenter of the body of 10 or more millimeters, compared to only five $(50 \%)$ of the ten male patients with OSA. Distribution of the severity of OSA cases and the connection with real barycenter distribution is shown in table 1. (Table 1) 
Table 1. Distribution of obstructive sleep apnea cases among the analyzed patients

\begin{tabular}{|l|c|c|c|c|}
\hline OSA severity & Male $\left(\mathbf{n}_{\mathbf{1}}\right)$ & $\begin{array}{c}\text { Distance from real } \\
\text { barycenter more than } \\
\mathbf{1 0} \mathbf{~ m m}\end{array}$ & Female $\left(\mathbf{n}_{\mathbf{2}}\right)$ & $\begin{array}{c}\text { Distance from real } \\
\text { barycenter more than } \\
\mathbf{1 0} \mathbf{~ m m}\end{array}$ \\
\hline Mild (AHI=5-15) & $2(20 \%)$ & $0(0 \%)$ & $2(50 \%)$ & $2(100 \%)$ \\
\hline Moderate (AHI=15-30) & $5(50 \%)$ & $2(40 \%)$ & $1(25 \%)$ & $1(100 \%)$ \\
\hline Severe (AHI>30) & $3(30 \%)$ & $3(100 \%)$ & $1(25 \%)$ & $1(100 \%)$ \\
\hline Total cases $(\mathbf{N})$ & $10(71,43 \%)$ & $5(50 \%)$ & $4(28,57 \%)$ & $4(100 \%)$ \\
\hline
\end{tabular}

\section{Conclusions}

All information gathered by the two integrated static and dynamic analysis systems, along with the postural analyzer, HSAT and dental analysis can be used to define therapeutic interventions needed, as well as to generate exercise programmes and other corrective measures, in an interdisciplinary collaboration between specialists. More examination modalities are planned to be included in future scientific publications, defining better study groups with different types of occlusion.

\section{Acknowledgments}

The members of the scientific team would like to express their deep thankfulness to the Academic management of Medical University - Varna and to the Academic management of the Faculty of Dental medicine and the University medical and dental center for the continuous support and the opportunities provided.

\section{References}

1. M. Milkov, M. Stoykov, H. Arnautska, S. Peev, D. Petrova. Important interrelations between posturology, vestibular disorders and dentistry. International bulletin of Otorhinolaryngology, Vol 16, (4), 2020, 29-32

2. Ambrosi F. Fondamenti di Posturologia. Ed. 2012

3. Oravitan M. Posturology-fundamental concepts and practical applications. Analele UVT-Seria EFS. 2009; 11: 61-9.

4. Barker V. Postura, Posizione e Movimento. Ed. Mediterranee. 1998

5. Carini F, Mazzola M, Fici C, Palmeri S, Messina M, Damiani P, Tomasello G. Posture and posturology, anatomical and physiological profiles: overview and current state of art. Acta Biomed. 2017 Apr 28; 88(1): 11-16. doi: 10.23750/abm.v88i1.5309. PMID: 28467328; PMCID: PMC6166197

6. https://www.ashlins.co.uk/therapies/posturology/

7. https://posturepro.co/why-posturology/

8. Kapandji IA. Fisiologia articolare, volume III. Monduzzi Editore. 2004

9. Autori vari. Fisologia dell'uomo, Edi. Eremes. 2002

10. Posturology. Methodological problems and scientific evidence. Gori L, Firenzuoli F Recenti Prog Med. 2005 Feb; 96(2):89-91.

11. B. Bricot. Total postural reprogramming. 4th edition, 2008.

12. Instruction book, Global postural system PL0800 Leonardo (GPS, Chinesport).

13. Maurer-Grubinger, C., Avaniadi, I., Adjami, F. et al. Systematic changes of the static upper body posture with a symmetric occlusion condition. BMC Musculoskelet Disord 21, 636 (2020). https://doi.org/10.1186/s12891-020-03655-x

14. Sofyanti, Ervina \& Trelia, Boel \& Sihombing, Anrice. (2020). The correlation between back posture and sagittal jaw position in adult orthodontic patients. Journal of Taibah University Medical Sciences. 10.1016/j.jtumed.2020.10.009.

15. Thanathornwong B, Jalayondeja W. Vibrotactile -Feedback Device for Postural Balance Among Malocclusion Patients. IEEE J Transl Eng Health Med. 2020 Apr 27; 8: 2100406. doi: 10.1109/JTEHM.2020.2990527. PMID: 32411542; PMCID: PMC7217290.

16. Jesús, Alvarez-Herms \& Cirer-Sastre, Rafel \& Julia, Sonia \& Burtscher, Martin \& Corbi, Francisco. (2019). The Influence of Dental Occlusion on Dynamic Balance and Muscular Tone. Frontiers in Physiology. 10. 10.3389/fphys.2019.01626.

17. Morize A, Kapoula Z. Reeducation of vergence dynamics improves postural control. Neurosci Lett. 2017 Aug 24;656:22-30. doi: 10.1016/j. neulet.2017.07.025. Epub 2017 Jul 17. PMID: 28729073.

18. Trovato, Francesca \& Roggio, Federico \& Szychlinska, Marta \& Borzì, Francesca \& Musumeci, Giuseppe. (2015). Clinical Kinesiology and Posturology Applied to a Group of Italian Students. A Morphological Observational Study. Journal of Functional Morphology and Kinesiology. 1. 16-29. 10.3390/jfmk1010016.

19. Silvestrini-Biavati A, Migliorati M, Demarziani E, Tecco S, Silvestrini-Biavati P, Polimeni A, Saccucci M. Clinical association between teeth malocclusions, wrong posture and ocular convergence disorders: an epidemiological investigation on primary school children. BMC Pediatr. 2013 Jan 23; 13: 12. doi: 10.1186/1471-2431-13-12. PMID: 23343244; PMCID: PMC3561080.

20. Hedayati Z, Paknahad M, Zorriasatine F. Comparison of natural head position in different anteroposterior malocclusions. J Dent (Tehran). 2013 May; 10(3): 210-20. PMID: 25512747; PMCID: PMC4264092.

21. Herold, G et. al. Internal diseases, part 1. Medical publishing house "Sharov", Sofia, 2011

22. Kayabasi S, Iriz A, Cayonu M, Cengiz B, Acar A, Boynuegri S, Mujdeci B, Eryilmaz A. Vestibular functions were found to be impaired in patients with moderate-to-severe obstructive sleep apnea. Laryngoscope. 2015 May; 125(5): 1244-8. doi: 10.1002/lary.25021. Epub 2014 Nov 12. PMID: 25388224. 
23. Gallina S, Dispenza F, Kulamarva G, Riggio F, Speciale R. Obstructive sleep apnoea syndrome (OSAS): effects on the vestibular system. Acta Otorhinolaryngol Ital. 2010; 30(6): 281-284.

24. M. Stoykov, M. Milkov. Impact of obstructive sleep apnea on posture and balance of the human body - a literature review, 4TH INTERNATIONAL HEALTH SCIENCES AND INNOVATION CONGRESS Proceedings book, Bacu, Azerbaijan, 4-5.07.2021, ISBN: 9781-955094-10-8, 278-295.

25. Lin CM, Davidson TM, Ancoli-Israel S. Gender differences in obstructive sleep apnea and treatment implications. Sleep Med Rev. 2008; 12(6): 481-496. doi:10.1016/j.smrv.2007.11.003

26. Young T. The occurrence of sleep-disordered breathing among middle-aged adults. N Engl J Med. 1993; 328: 1230-1235

27. Quintana-Gallego E, Carmon-Bernal C, Capote F, et al. Gender differences in obstructive sleep apnea syndrome: a clinical study of 1166 patients. Respir Med. 2004; 98: 984-989.

28. (Сергеева, М., 2015)

29. Olmos SR, Kritz-Silverstein D, Halligan W, Silverstein ST. The effect of condyle fossa relationships on head posture. Cranio. $2005 ; 23: 48-52$.

30. D'Attilio M, Epifania E, Ciuffolo F, Salini V, Filippi MR, Dolci M, et al. Cervical lordosis angle measured on lateral cephalograms; findings in skeletal class II female subjects with and without TMD: a cross sectional study. Cranio. 2004; 22: 27-44.

31. Gamlin PD. Neural mechanisms for the control of vergence eye movements. Ann N Y Acad Sci. 2002 Apr; 956: 264-72. doi: 10.1111/j.17496632.2002.tb02825.x. PMID: 11960810.

32. Serra A, Chen AL, Leigh RJ. Disorders of vergence eye movements. Curr Opin Neurol. 2011 Feb; 24(1): 32-7. doi: 10.1097/ WCO.0b013e328341eebd. PMID: 21150598

Reviewer of the article: Prof. Dr. Augusto Pietro Casani, Professor in the Otolaryngology Sections at the University of Pisa

\section{Correspondence to:}

Dr. Miroslav Stoykov, DMD

Department of Dental materials science and propaedeutics of prosthetic dental medicine

Faculty of Dental medicine, Medical University - Varna

+359896787758

miroslavstoikov93@abv.bg 